\title{
Évaluation de la vulnérabilité à la pollution potentielle de la nappe côtière alluvionnaire de Meltine-Ras Jebel-Raf Raf (Nord-Est tunisien) selon les méthodes paramétriques DRASTIC, SINTACS et SI
}

\section{A GIS-based assessment of the vulnerability to potential pollution of the alluvial aquifer of Meltine-Ras Jebel-Raf Raf (Northeats coast of Tunisia) using the parametric methods DRASTIC, SINTACS and SI}

\author{
Mohamed Hafedh Hamza, Ayed Added, Alain Francès, Ramiro Rodriguez, \\ Mohamed Ajmi et Saâdi Abdeljaoued
}

Volume 21, numéro 1, 2008

URI : https://id.erudit.org/iderudit/017932ar

DOI : https://doi.org/10.7202/017932ar

Aller au sommaire du numéro

Éditeur(s)

Université du Québec - INRS-Eau, Terre et Environnement (INRS-ETE)

ISSN

1718-8598 (numérique)

Découvrir la revue

Citer cet article

Hamza, M. H., Added, A., Francès, A., Rodriguez, R., Ajmi, M. \& Abdeljaoued, S. (2008). Évaluation de la vulnérabilité à la pollution potentielle de la nappe côtière alluvionnaire de Meltine-Ras Jebel-Raf Raf (Nord-Est tunisien) selon les méthodes paramétriques DRASTIC, SINTACS et SI. Revue des sciences de l'eau / Journal of Water Science, 21(1), 75-86. https://doi.org/10.7202/017932ar

\section{Résumé de l'article}

La nappe alluvionnaire de Metline-Ras Jebel-Raf Raf (gouvernorat de Bizerte, côte Nord Est de la Tunisie), qui occupe une superficie de $35 \mathrm{~km}^{2}$, représente une ressource économique jugée prioritaire du fait qu'elle est utilisée dans les domaines d'irrigation et de consommation domestique. L'aire de la nappe est occupée essentiellement par des zones agricoles caractérisées par une utilisation de plus en plus importante des engrais chimiques qui représentent, en plus des rejets des zones industrielles, un risque permanent pour la qualité des eaux souterraines. L'étude de la vulnérabilité à la pollution par les polluants inorganiques de cette nappe a été effectuée en appliquant les méthodes DRASTIC standard (Aller et al., 1987; Engel et al., 1996), SINTACS (Civita, 1994) et SI (Ribeiro, 2000), avec l'aide des logiciels des systèmes d'information géographique, ARC/Info et Idrisi. Une comparaison statistique des cartes de vulnérabilité obtenues a été effectuée. Cette comparaison a montré une certaine ressemblance entre les résultats obtenus en utilisant les méthodes SINTACS et SI. En revanche, la carte DRASTIC standard s'avère différente par rapport aux autres. 
ÉVALUATION DE LA VULNÉRABILITÉ À LA POLLUTION POTENTIELLE DE LA NAPPE CÔTIÈRE ALLUVIONNAIRE DE MELTINE-RAS JEBEL-RAF RAF (NORD-EST TUNISIEN) SELON LES MÉTHODES PARAMÉTRIQUES DRASTIC, SINTACS ET SI

A GIS-based assessment of the vulnerability to potential pollution of the alluvial aquifer of Meltine-Ras Jebel-Raf Raf (Northeats coast of Tunisia) using the parametric methods DRASTIC, SINTACS and SI

Mohamed Hafedh HAMZA ${ }^{1}$, Ayed ADDED ${ }^{1}$, Alain FRANCÈS ${ }^{2}$, Ramiro RODRIGUEZ ${ }^{3}$, Mohamed AJMI $^{1}$, Saâdi ABDELJAOUED ${ }^{1}$

${ }^{1}$ Université de Tunis El Manar, Faculté des Sciences de Tunisie, Département de Géologie, Laboratoire des Ressources Minérales et Environnement, 20092, El Manar II, Tunis, Tunisie

${ }^{2}$ Instituto Nacional de Engenharia, Technologia e Inovação (INETI), Dpt Hidrogeologia,

Estrada Portela Zambujal Alfragide, Apartado 7586, 2720-866, Amadoram, Portugal

${ }^{3}$ Universidad Nacional Autonoma de México, Instituto de Geofisica, Dpt de Recursos Minerales Ciudad Universitaria, 04510 México, D.F., México

Reçu le 15 mars 2006, accepté le 31 janvier 2008

\section{RÉSUMÉ}

La nappe alluvionnaire de Metline-Ras Jebel-Raf Raf (gouvernorat de Bizerte, côte Nord Est de la Tunisie), qui occupe une superficie de $35 \mathrm{~km}^{2}$, représente une ressource économique jugée prioritaire du fait qu'elle est utilisée dans les domaines d'irrigation et de consommation domestique. L'aire de la nappe est occupée essentiellement par des zones agricoles caractérisées par une utilisation de plus en plus importante des engrais chimiques qui représentent, en plus des rejets des zones industrielles, un risque permanent pour la qualité des eaux souterraines. L'étude de la vulnérabilité à la pollution par les polluants inorganiques de cette nappe a été effectuée en appliquant les méthodes DRASTIC standard (Aller et al., 1987;
Engel et al., 1996), SINTACS (Civita, 1994) et SI (Ribeiro, 2000), avec l'aide des logiciels des systèmes d'information géographique, ARC/Info et Idrisi. Une comparaison statistique des cartes de vulnérabilité obtenues a été effectuée. Cette comparaison a montré une certaine ressemblance entre les résultats obtenus en utilisant les méthodes SINTACS et SI. En revanche, la carte DRASTIC standard s'avère différente par rapport aux autres.

Mots clés : Vulnérabilité, pollution, nappe alluvionnaire, polluants inorganiques, DRASTIC, SINTACS, SI, SIG, comparaison statistique.

*Auteur pour correspondance :

Téléphone: 21671872600

Télécopie : 21671885408

Courriel : hafedh.hamza@fst.mu.tn 


\section{ABSTRACT}

The alluvial aquifer of Metline-Ras Jebel-Raf Raf (prefecture of Bizerta, northeast coast of Tunisia), which occupies a surface of $35 \mathrm{~km}^{2}$, is of great economic importance because it is used for irrigation and domestic consumption. The area of the aquifer is essentially occupied by agricultural zones characterised by an increasing use of chemical fertilizers. These chemical fertilizers, together with discharges from the industrial zones that have appeared during the last 25 years, threaten the quality of the ground waters. The vulnerability of the aquifer to pollution by generic pollutants has been studied by applying the following methods: the generic DRASTIC method, the SINTACS method and the SI method. For the application of these methods GIS techniques were used. A statistical comparison among the vulnerability maps generated by each method has been carried out. On the one hand, this comparison shows a certain similarity between the results obtained using the SI and SINTACS methods. On the other hand, the DRASTIC map classification shows different results.

Keywords: Vulnerability, pollution, alluvial aquifer, generic pollutants, DRASTIC, SINTACS, SI, GIS, statistical comparison.

\section{INTRODUCTION}

Les eaux souterraines représentent une importante ressource exploitée pour la consommation humaine et pour l'utilisation dans les domaines agricoles et industriels. Ces eaux sont souvent menacées par la contamination par des polluants de différente nature : biologique, chimique ou physique. La prévention contre la pollution des nappes constitue une étape importante à laquelle les scientifiques consentent de plus en plus d'effort, notamment en étudiant la vulnérabilité des nappes souterraines. La notion de vulnérabilité à la pollution d'un aquifère est définie comme sa susceptibilité intrinsèque à la modification de la qualité et de la quantité d'eau souterraine dans l'espace et dans le temps, à cause des processus naturels et/ou de l'activité anthropique (CIVITA, 1994). La nappe étudiée est la nappe phréatique de Metline-Ras Jebel-Raf Raf située dans le gouvernorat de Bizerte au Nord-Est de la Tunisie (Figure 1). Cette nappe est localisée dans une plaine côtière essentiellement exploitée par l'agriculture et où de plus en plus d'installations industrielles s'implantent. Ainsi, ce travail a été réalisé dans le but d'établir des cartes de vulnérabilité à la pollution par les polluants inorganiques suivant les méthodes paramétriques suivantes: la méthode DRASTIC standard (ALLER et al., 1987) avec sa classification classique et sa classification proposée par ENGEL et al. (1996), la méthode SINTACS (CIVITA, 1994), et la méthode SI (RIBEIRO, 2000). D'autre part, une comparaison statistique des résultats obtenus par les différentes méthodes, a été effectuée.

Les méthodes utilisées se présentent sous forme de systèmes de cotation numérique basés sur la considération des différents facteurs influençant le système hydrogéologique. Les méthodes DRASTIC et SINTACS prennent en compte les sept paramètres suivants : la profondeur de la nappe, la recharge efficace de l'aquifère, la lithologie de l'aquifère, les types de sol, la topographie, l'impact de la zone vadose ou l'effet de l'autoépuration de la zone vadose, et la conductivité hydraulique de l'aquifère. En revanche, la méthode SI prend en compte les seuls cinq paramètres suivants : la profondeur de la nappe, la recharge efficace de l'aquifère, les types de sol, la topographie et l'occupation des sols.

\section{METHODES D'ÉTUDE}

\subsection{La méthode DRASTIC}

La méthode DRASTIC, développée par les services de l'Agence américaine de protection de l'environnement USEPA (ALLER et al., 1987), est une méthode d'évaluation de la vulnérabilité intrinsèque verticale à la pollution des aquifères par systèmes paramétriques; le principe commun de ces systèmes consiste à sélectionner préalablement les paramètres sur lesquels se base l'évaluation de la vulnérabilité. Chaque paramètre est subdivisé en intervalles de valeurs significatives et affecté d'une cotation numérique croissante en fonction de son importance dans la vulnérabilité. L'acronyme DRASTIC correspond aux initiales des sept facteurs déterminant la valeur de l'indice de vulnérabilité : Depth to water (D) : profondeur de la nappe; Net Recharge (R) : recharge efficace de l'aquifère; Aquifer media (A) : la lithologie de l'aquifère; Soil media (S) : type de sol; Topography (T) : pente topographique du terrain; Impact of vadose zone (I) : impact de la zone vadose (zone non saturée); Hydraulic Conductivity of the aquifer (C) : conductivité hydraulique de l'aquifere. Les sept paramètres découpent, de façon schématique, une unité hydrogéologique locale en ses principales composantes, lesquelles influencent à différents degrés les processus de transport et d'atténuation des contaminants dans le sol, ainsi que leur temps de transport. Une valeur numérique appelée poids paramétrique, comprise entre 1 et 5 , est attribuée à chaque paramètre, reflétant son degré d'influence. Chaque paramètre est classé en classes associées à des cotes variant de 1 à 10 . La plus petite cote représente les conditions de plus faible vulnérabilité à la contamination. Une valeur numérique appelée indice de vulnérabilité DRASTIC et notée ID est déterminée : elle décrit le degré de vulnérabilité 


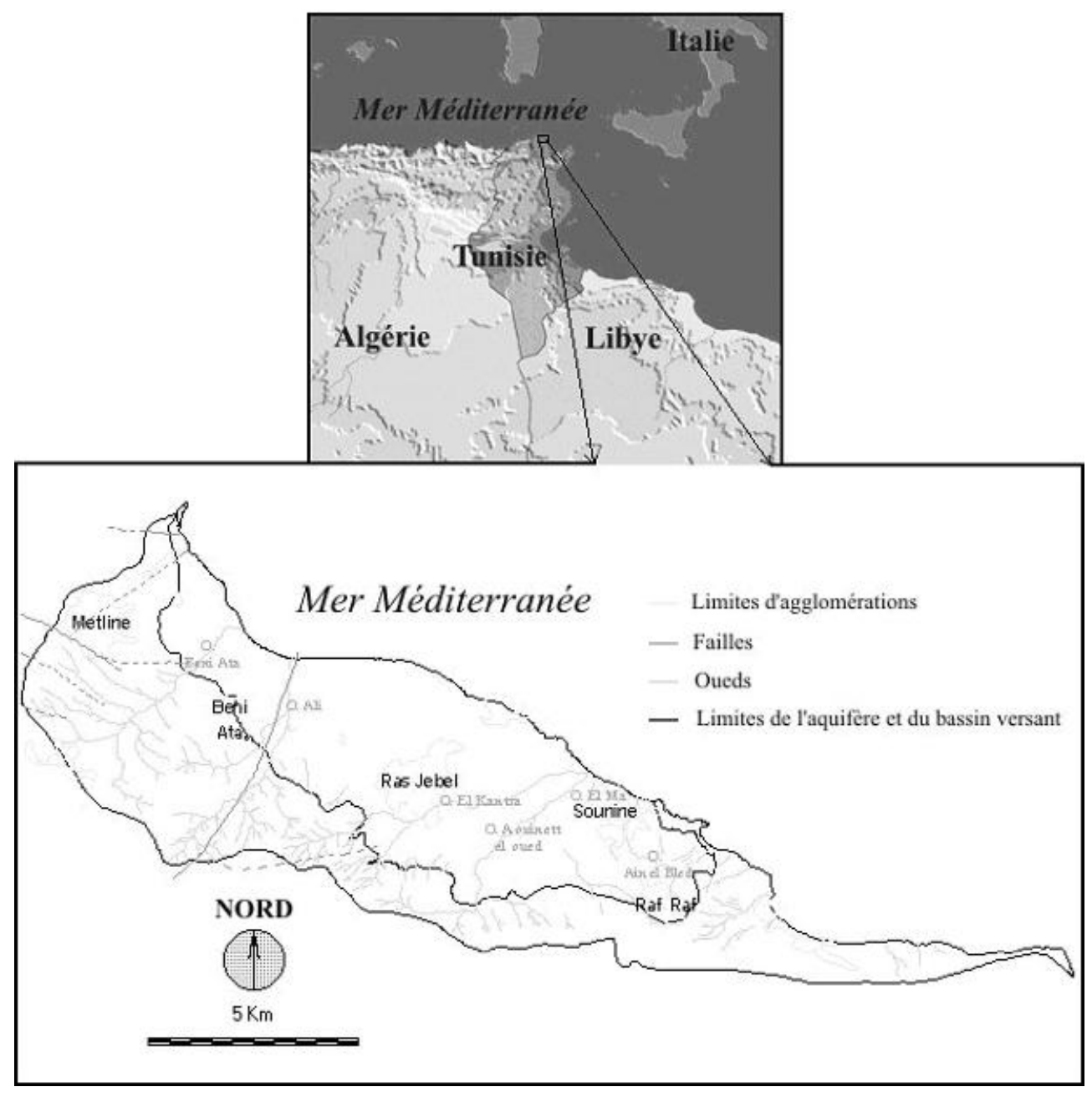

Figure 1 : La nappe alluvionnaire de Metline-Ras Jebel-Raf Raf. The alluvial aquifer of Metline-Ras Jebel-Raf Raf

de chaque unité hydrogéologique. L'indice de vulnérabilité DRASTIC est calculé en faisant la somme des produits des cotes par les poids des paramètres correspondants :

$$
\begin{aligned}
& \mathrm{ID}=\mathrm{Dp}^{*} \mathrm{Dc}+\mathrm{Rp}^{*} \mathrm{Rc}+\mathrm{Ap}^{*} A \mathrm{c}+\mathrm{Sp}^{*} \mathrm{Sc}+\mathrm{Tp}^{*} \mathrm{Tc} \\
& +\mathrm{Ip}^{*} \mathrm{Ic}+\mathrm{Cp}^{*} \mathrm{Cc}
\end{aligned}
$$

(où $\mathrm{D}, \mathrm{R}, \mathrm{A}, \mathrm{S}, \mathrm{T}$, I, et $\mathrm{C}$ les sept paramètres de la méthode DRASTIC, $\mathrm{p}$ étant le poids du paramètre et $\mathrm{c}$, la cote associée).

Il existe deux versions de la méthode DRASTIC : la version DRASTIC standard, appliquée dans le cas où les contaminants considérés sont des polluants inorganiques (cas de notre article), et la version DRASTIC pesticides, appliquée dans le cas où les contaminants considérés sont des pesticides. Les valeurs des poids des paramètres, dans la version standard de la méthode DRASTIC, sont présentés dans le tableau 1.
Les valeurs de l'indice DRASTIC obtenues représentent la mesure de la vulnérabilité hydrogéologique de l'aquifère, elles varient de 23 à 226 dans le cas de la version standard. Les valeurs obtenues sont regroupées, selon ALLER et al. (1987), en cinq classes dont chacune correspond à un degré de vulnérabilité (Tableau 2). D’autre part, ENGEL et al. (1996) propose la classification des valeurs en quatre autres classes (Tableau 3).

\subsection{La méthode SINTACS}

La méthode SINTACS (CIVITA, 1994) est la version italienne de la méthode DRASTIC : c'est une adaptation de cette méthode aux conditions méditerranéennes. Cette méthode prend en considération les mêmes paramètres que la méthode DRASTIC avec des poids et des cotes différentes. L'acronyme SINTACS correspond aux initiales des sept facteurs suivants : la 
Tableau 1 : Poids des paramètres dans la version standard de la méthode DRASTIC.

Table 1: Generic DRASTIC parameter weights.

\begin{tabular}{cc}
\hline Paramètre & $\begin{array}{c}\text { Version DRASTIC } \\
\text { standard }\end{array}$ \\
\hline D : profondeur de la nappe & 5 \\
$\mathbf{R}:$ recharge efficace & 4 \\
$\mathbf{A}:$ matériaux de l'aquifère & 3 \\
$\mathbf{S}:$ type de sol & 2 \\
$\mathbf{T}:$ pente du terrain & 1 \\
$\mathbf{I}:$ matériaux de la zone non saturée & 5 \\
$\mathbf{C}:$ conductivité hydraulique de l'aquifère & 3 \\
\hline
\end{tabular}

Tableau 2 : Critères d'évaluation de la vulnérabilité dans la méthode DRASTIC (ALLER et al., 1987.

Table 2: Criteria for the evaluation of vulnerability in the DRASTIC method (ALLER et al., 1987).

\begin{tabular}{cc}
\hline Degré de vulnérabilité & Indice de vulnérabilité \\
\hline Très faible & $<80$ \\
Faible & $80-120$ \\
Moyen & $121-160$ \\
Élevé & $161-200$ \\
Très élevé & $>200$ \\
\hline
\end{tabular}

Tableau 3 : Critères d'évaluation de la vulnérabilité dans la méthode DRASTIC (ENGEL et al., 1996).

Table 3: Criteria for the evaluation of vulnerability in the DRASTIC method (ENGEL et al., 1987).

\begin{tabular}{cc}
\hline Degré de vulnérabilité & Indice de vulnérabilité \\
\hline Faible & $<101$ \\
Moyen & $101-140$ \\
Élevé & $141-200$ \\
Très élevé & $>200$ \\
\hline
\end{tabular}

profondeur du plan d'eau ( $S$ = Soggiacenzia), la recharge efficace de l'aquifère (I = infiltrazione), l'effet de l'autoépuration de la zone vadose ( $\mathrm{N}$ = effeto di autoepurazione del non-saturo), le type de sol ( $\mathrm{T}$ = typologia della copertura), les caractéristiques hydrogéologiques de l'aquifère (A = caratteristiche idrogeologische dell'acquifero), la conductivité hydraulique de l'aquifère $(\mathrm{C}=$ conductibilità dell'acquifero $)$ et la pente topographique ( $\mathrm{S}=$ l'acclivita della superficie topografica).

La spécificité de cette méthode consiste au fait qu’elle propose cinq scénarios différents :

- Scénario «Impact Normal»: scénario relatif aux aquifères constitués par des sédiments non consolidés avec une profondeur de la nappe qui n'est pas très élevée, localisés dans des aires à sols épais. Les zones relatives à ce scénario correspondent aux régions stables, de point de vue occupation des sols, avec existence ou non de terres cultivées, une utilisation faible de pesticides, de fertilisants et d'irrigation, et des périmètres urbains très dispersés;

- Scénario "Impact Sévère " : correspond aux mêmes types d'aquifères soumis à une occupation des sols intensive, avec des terres cultivées à forte utilisation de pesticides, de fertilisants et d'irrigation, des implantations industrielles et urbaines denses, et des dépôts liquides et solides de déchets;

- Scénario «Drainage important à partir d'un réseau superficiel " : scénario relatif aux aires où il y a une forte infiltration vers l'aquifère à partir d'un réseau superficiel d'eau; 
- Scénario "Terrain très karstifié ";

- Scénario «Terrain fissuré".

Les poids attribués aux différents paramètres dans les différentes versions SINTACS sont présentés dans le tableau 4. Quatre classes de vulnérabilité peuvent êtres identifiées selon les valeurs des indices de vulnérabilité (Tableau 5).

\subsection{La méthode SI (Susceptibility Index)}

La méthode SI (Susceptibility Index ou méthode d'Indice de Susceptibilité), qui estime la vulnérabilité verticale spécifique à la pollution agricole (essentiellement par les nitrates et également par les pesticides), a été développée au Portugal par RIBEIRO (2000). Cette méthode prend en considération cinq paramètres. Les quatre premiers paramètres sont identiques à quatre paramètres déjà utilisés dans la méthode DRASTIC (D:la profondeur de la nappe, $\mathrm{R}$ : la recharge efficace de l'aquifère, $\mathrm{A}:$ la lithologie de l'aquifere, et $\mathrm{T}$ : la pente topographique du terrain). Les cotes correspondantes aux différentes classes de ces paramètres, dans la méthode DRASTIC, ont été également conservées. Un cinquième nouveau paramètre a été introduit : le paramètre occupation des sols (OS). La classification utilisée pour ce paramètre est la classification CORINE Land Cover (EUROPEAN COMMUNITY, 1993) (Tableau 6). Une valeur appelée facteur d'occupation des sols et notée LU, variant de 0 à 100 , est attribuée à chaque classe d'occupation des sols. Il est à signaler que les valeurs des cotes attribuées aux classes des différents paramètres ont été multipliées par 10 pour faciliter la lecture des résultats obtenus. Ces valeurs varient par conséquent de 0 à 100 , allant du moins vulnérable au plus vulnérable. Les poids attribués aux paramètres SI varient de 0 à 1 selon l'importance du paramètre dans la vulnérabilité (Tableau 7).

La méthode SI présente quatre degrés de vulnérabilité selon les valeurs des indices obtenues (Tableau 8).

Il est à signaler que les paramètres, qui ne sont pas pris en compte dans la méthode SI par rapport à la méthode

Tableau 4 : Poids attribués aux paramètres SINTACS dans les différents scénarios de la méthode. Table 4: Weights attributed to parameters in the different scenarios of the SINTACS method.

\begin{tabular}{cccccc}
\hline $\begin{array}{c}\text { Scénario } \\
\text { Paramètre }\end{array}$ & $\begin{array}{c}\text { Impact } \\
\text { Normal }\end{array}$ & $\begin{array}{c}\text { Impact } \\
\text { Sévère }\end{array}$ & $\begin{array}{c}\text { Drainage } \\
\text { Important }\end{array}$ & Karst & Terrains Fissurés \\
\hline S & 5 & 5 & 4 & 2 & 3 \\
I & 4 & 5 & 4 & 5 & 3 \\
$\mathbf{N}$ & 5 & 4 & 4 & 1 & 3 \\
$\mathbf{T}$ & 4 & 5 & 2 & 3 & 4 \\
$\mathbf{A}$ & 3 & 3 & 5 & 5 & 4 \\
$\mathbf{C}$ & 3 & 2 & 5 & 5 & 5 \\
S & 2 & 2 & 2 & 5 & 4 \\
\hline
\end{tabular}

Tableau 5: Critères d'évaluation de la vulnérabilité dans la méthode SINTACS.

Table 5: $\quad$ Criteria for the evaluation of vulnerability in the SINTACS method.

\begin{tabular}{cc}
\hline Degré de vulnérabilité & Indice de vulnérabilité \\
\hline Faible & $<106$ \\
Moyen & $106-186$ \\
Élevé & $187-210$ \\
Très élevé & $>210$ \\
\hline
\end{tabular}


Tableau 6: Principales classes d'occupation des sols et valeurs correspondantes.

Table 6: $\quad$ Main soil occupation classes and corresponding values.

\begin{tabular}{lc}
\hline \multicolumn{1}{c}{$\begin{array}{c}\text { Occupation des sols selon la classification } \\
\text { CORINE Land Cover }\end{array}$} & $\begin{array}{c}\text { Valeur du facteur } \\
\text { d'occupation des sols LU (Land use factor) }\end{array}$ \\
\hline Décharge industrielle, décharge d'ordures, mines & 100 \\
Périmètres irrigués, rizières & 90 \\
Carrière, chantier naval & 80 \\
Zones artificielles couvertes, zones vertes & 75 \\
Cultures permanentes (vignes, vergers, oliviers, etc.) & 70 \\
Zones urbaines discontinues & 70 \\
Pâturages et zones agro-forestières. & 50 \\
Milieux aquatiques (marais, salines, etc.) & 50 \\
Forêts et zones semi-naturelles & 0 \\
\hline
\end{tabular}

Tableau 7 : Poids attribués aux paramètres SI.

Table 7: $\quad$ Weights attributed to SI parameters.

\begin{tabular}{lccccc}
\hline PARAMÈTRE & $\mathrm{D}$ & $\mathrm{R}$ & $\mathrm{A}$ & $\mathrm{T}$ & $\mathrm{OS}$ \\
\hline POIDS & 0,186 & 0,212 & 0,259 & 0,121 & 0,222 \\
\hline
\end{tabular}

Tableau 8 : Critères d'évaluation de la vulnérabilité dans la méthode SI.

Table 8: $\quad$ Criteria for the evaluation of vulnerability in the SI method.

\begin{tabular}{cc}
\hline Degré de vulnérabilité & Indice de vulnérabilité \\
\hline Faible & $<45$ \\
Moyen & $45-64$ \\
Élevé & $65-84$ \\
Très élevé & $85-100$ \\
\hline
\end{tabular}

DRASTIC sont les suivants: conductivité hydraulique de l'aquifere, impact de la zone vadose et type de sol. En effet, la méthode SI considère que le paramètre conductivité hydraulique de l'aquifère est difficile à évaluer dans l'espace. De plus, ce dernier paramètre a été déjà pris en compte indirectement dans le paramètre A (lithologie de l'aquifère) par la considération des caractéristiques granulométriques de l'aquifère. RIBEIRO (2000) minimise également le rôle de la zone vadose en se basant sur les travaux de FOSTER (1987), et ceux de VRBA et ZOPOROZEC (1994) qui considèrent également que les processus d'atténuation relatifs au paramètre type de sol sont sans grand effet sur la vulnérabilité, bien que ce paramètre soit indirectement pris en considération à travers le paramètre occupation des sols.

Plusieurs applications de cette méthode ont été effectuées (BATISTA, 2004; FRANCES et al., 2002; OLIVEIRA et
LOBO FERREIRA, 2005; STIGTER et al., 2006). Ces études ont montré une bonne corrélation entre les zones considérées comme vulnérables par cette méthode et les zones réellement contaminées.

\section{SITE D'ÉTUDE}

La zone de Metline-Ras Jebel-Raf Raf est une plaine côtière située dans le Nord-Est tunisien entre les latitudes 514.411 et 526.215 Nord et les longitudes 431.657 et 440.351 Est (UTM Ref) (Figure 1). Le bassin hydrogéologique occupe une superficie de $50 \mathrm{~km}^{2}$ avec une population d'environ 55000 habitants. Les hauteurs des bordures montagneuses excèdent rarement $300 \mathrm{~m}$. La nappe alluvionnaire couvre environ $35 \mathrm{~km}^{2}$ et elle est située approximativement entre les 
altitudes 0 et $50 \mathrm{~m}$. Les principales villes et villages du bassin versant sont Metline, Ras Jebel, Raf Raf, Beni Ata et Sounine. La pluviométrie annuelle varie, de $495 \mathrm{~mm}$ à $638 \mathrm{~mm}$. Plus de 200 puits sont actuellement exploités au niveau de la nappe avec un volume moyen de 1,5 millions de $\mathrm{m}^{3}$ d'eau extraite annuellement. Les ressources de cette nappe sont menacées par l'utilisation de plus en plus accentuée d'engrais chimiques dans le domaine agricole, ainsi que par les rejets domestiques en l'absence de stations d'épuration fonctionnelles dans la région. Les rejets de la zone industrielle de Ras Jebel représentent également une grande menace pour la nappe.

\section{APPLICATION DES MÉTHODES DRASTIC, SINTACS ET SI}

\subsection{Données utilisées}

L'identification des unités et des sous-unités hydrogéologiques ainsi que l'évaluation des différents paramètres considérés dans les méthodes DRASTIC, SINTACS et SI, requièrent une bonne connaissance de la géologie, de l'hydrogéologie, de la pédologie, de la topographie ainsi que de la météorologie. Ainsi, les travaux effectués dans la zone d'études dansles domaines climatiques (INM, 1991-2004), géologiques (BUROLLET, 1951; EL GHALI et BEN AYED, 2000), géophysiques (ESSAYEH, 1996), hydrogéologiques (DGRE, 1991-2004; ENNABLI, 1969), pédologiques (ASB, 1997-2000; FOURNET et MOURI, 1990; MANSOUR, 1988), topographiques (OTC, 1981), d'occupation des sols (CRDA de Bizerte, 1999), ont permis d'identifier ces différents paramètres pour l'ensemble du territoire étudié. Pour le paramètre profondeur de la nappe, une interpolation des valeurs de profondeur de la nappe enregistrées en 2004 sur plus de 60 puits bien répartis sur la région d'étude a été faite, puis une carte piézométrique a été tracée; pour le paramètre recharge efficace de l'aquifère, il a été calculé grâce à l'équation suivante : $\mathrm{I}=\mathrm{P}$. c $(\mathrm{mm} / \mathrm{an})($ ENGLAND, 1973) où $\mathrm{I}:$ la recharge efficace annuelle de l'aquifère $(\mathrm{mm}), \mathrm{P}$ : la pluviométrie annuelle $(\mathrm{mm})$, et $\mathrm{c}$ : le coefficient d'infiltration potentielle qui dépend de la nature du sol. Cette équation est proposée par CIVITA (1994) dans le cas des sols ayant une épaisseur dépassant $0,5 \mathrm{~m}$, comme c'est le cas pour les sols présents dans notre zone d'étude. Les données relatives à la lithologie de la zone non saturée ont été déterminées en utilisant la méthode de corrélation sur la base de la lithologie des forages disponibles et la nature des terrains d'après la carte géologique. Les données relatives au type de sol ont été extraites de la carte pédologique couvrant une grande partie de la zone d'étude, ainsi qu’à partir de quelques études pédologiques ponctuelles effectuées dans la région. La lithologie de l'aquifère a été déterminée en se basant sur les données des forages effectués par ENNABLI en 1969 et sur les données de profondeur de la nappe en 2004 (les épaisseurs de la zone saturée ont été ajustées en fonction de la variation du niveau de la nappe). La conductivité hydraulique de l'aquifère a été calculée en utilisant les données de transmissivité et les données de l'épaisseur de l'aquifère. Enfin, la pente topographique du terrain a été calculée en utilisant les trois cartes topographiques couvrant la région d'étude.

Les systèmes d'information géographique (SIG) représentent l'outil le plus adéquat pour l'application de ces méthodes paramétriques. Les logiciels ARC/Info et Idrisi ont été utilisés dans le présent travail.

\subsection{Application de la méthode DRASTIC standard}

La méthode DRASTIC a été déjà appliquée dans sa version pesticides (HAMZA et al., 2004) et dans sa version standard (HAMZA et al., 2006) dans la zone d'étude. Les deux versions de cette méthode diffèrent par les valeurs des poids attribués aux paramètres «type de sol», "pente topographique du terrain» et «impact de la zone vadose». La carte de vulnérabilité DRASTIC standard basée sur la classification d'ALLER et al. (1987) (Figure 2) montre l'existence de trois degrés de vulnérabilité dans la zone d'étude : très faible, faible et moyen. Les zones à très faible vulnérabilité occupent $16 \%$ de la surface totale, celles à faible vulnérabilité occupent $61 \%$, et celles à vulnérabilité moyenne n'occupent que $23 \%$. D'autre part, la carte de vulnérabilité DRASTIC standard basée sur la classification d'ENGEL et al. (1996) montre l'existence des degrés de vulnérabilité suivants: faible, moyen et élevé (Figure 3) avec $46 \%$ des territoires à vulnérabilité faible, $48 \%$ à vulnérabilité moyenne et $6 \%$ à vulnérabilité élevée. Il est à noter que pour pouvoir faire une comparaison entre la carte de vulnérabilité DRASTIC classée selon ALLER et al. (1987) et les autres méthodes, nous avons regroupé les classes à très faible et à faible vulnérabilité sous le terme «vulnérabilité faible».

\subsection{Application de la méthode SINTACS}

Pour les paramètres quantitatifs de la méthode SINTACS (profondeur de la nappe, recharge efficace, conductivité hydraulique de l'aquifere et pente topographique), les classes se présentent sous forme d'intervalles de valeurs numériques, 


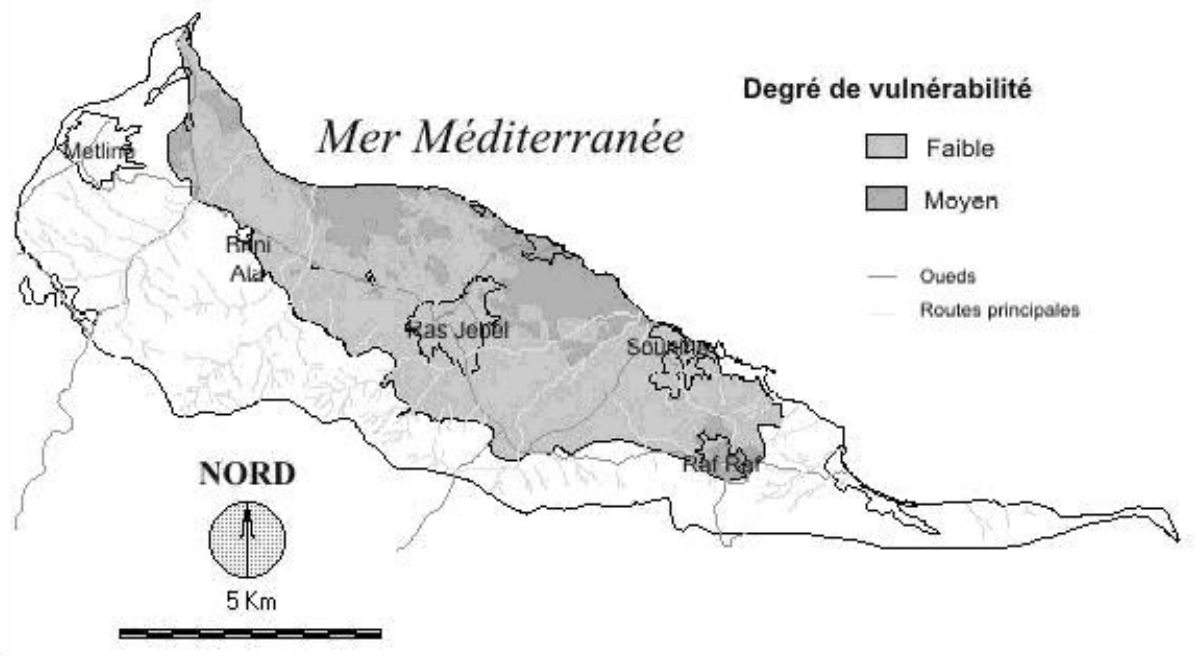

Figure 2 : Carte de vulnérabilité DRASTIC standard (classification d'ALLER et al., 1987). Generic DRASTIC vulnerability map (classification of ALLER et al., 1987).

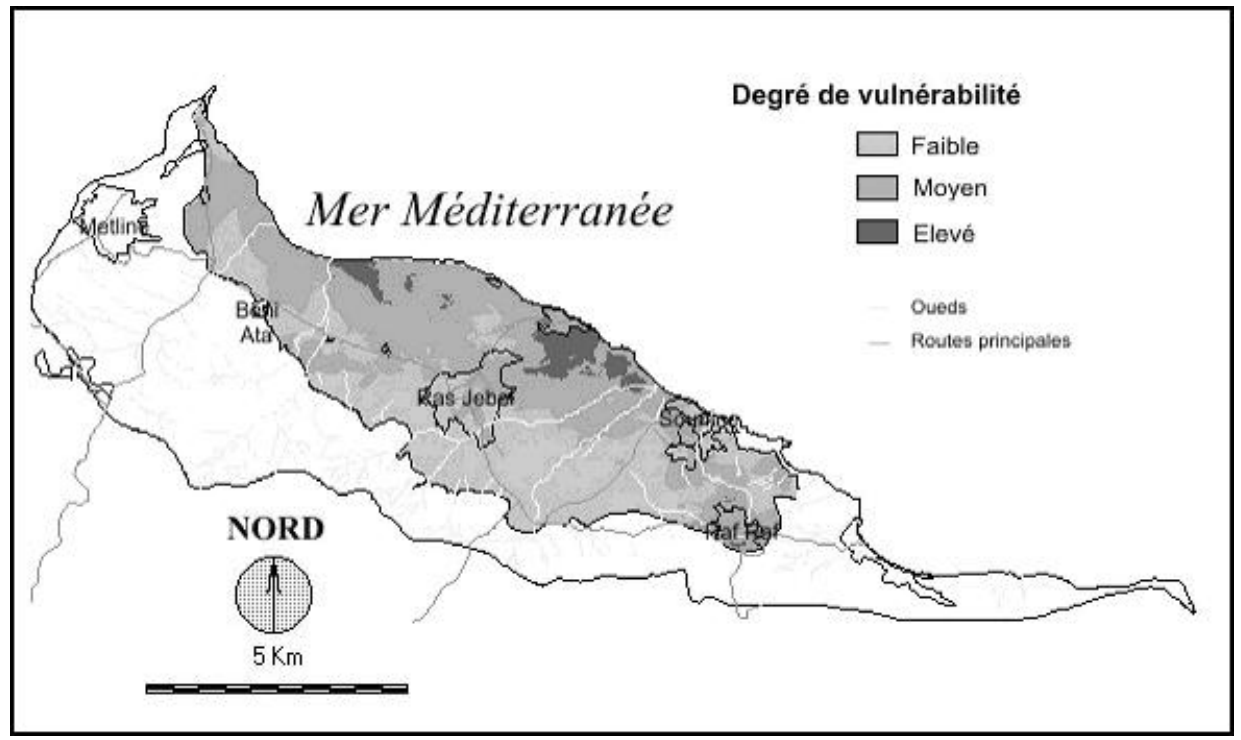

Figure 3 : Carte de vulnérabilité DRASTIC standard (classification d'ENGEL et al., 1996).

Generic DRASTIC vulnerability map (classification of ENGEL et al., 1996).

et ne posent pas, de ce fait, de problèmes dans la classification et dans l'attribution des cotes correspondantes.

Par contre, les paramètres qualitatifs (effet de l'autoépuration de la zone vadose, type de sol et lithologie de l'aquiferre) peuvent ne pas correspondre exactement aux classes proposées par les tableaux du guide pratique de la méthode SINTACS. Il faut alors suivre un raisonnement logique, pour pouvoir identifier les classes adéquates, et attribuer par la suite à chaque classe la cote correspondante.

La zone d'étude se subdivise en deux parties dont l'une coïncide avec le scénario «Impact Normal» et l'autre avec le scénario «Impact Sévère» de la méthode SINTACS. La carte de vulnérabilité finale obtenue (Figure 4), montre l'existence de 3 degrés de vulnérabilité : faible, moyen, et élevé. Les zones à faible vulnérabilité occupent $19 \%$ de la surface totale de la nappe, celles à vulnérabilité moyenne occupent $80 \%$, et celles à vulnérabilité élevée n’occupent que $1 \%$. 


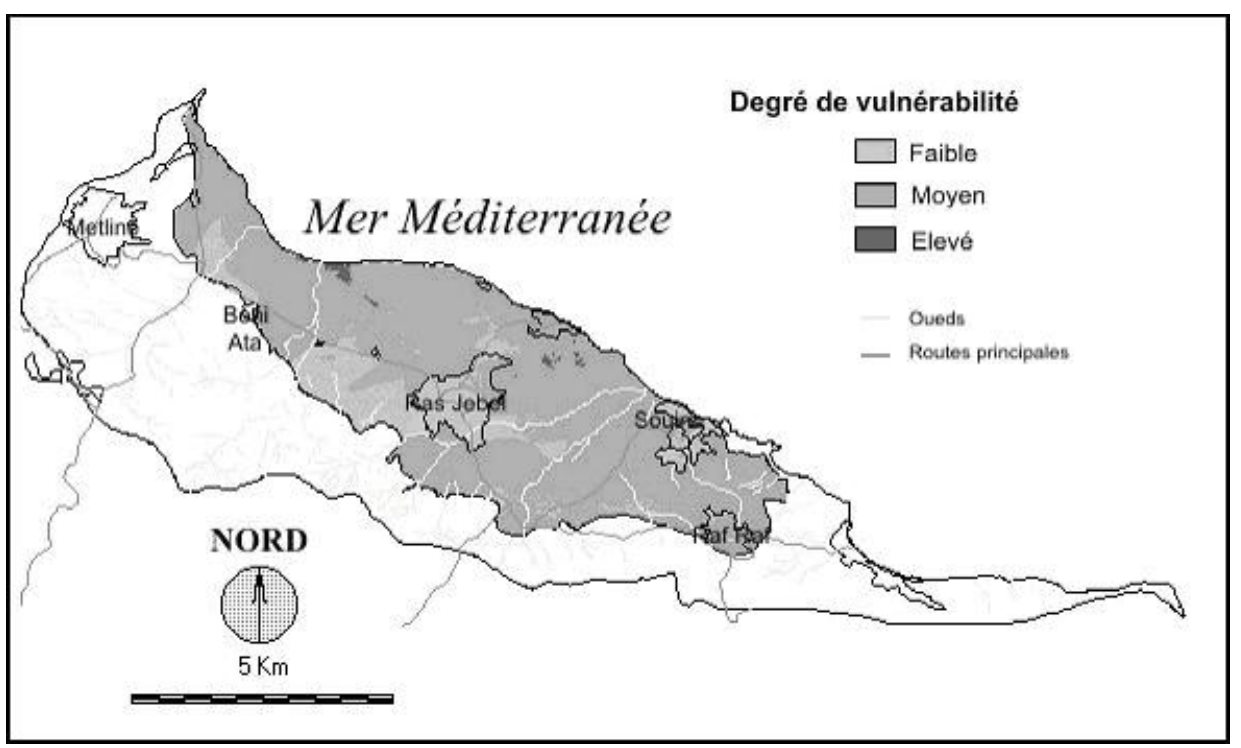

Figure 4 : Carte de vulnérabilité SINTACS. SINTACS vulnerability map.

\subsection{Application de la méthode SI}

Les cartes relatives aux quatre premiers paramètres nécessaires $(\mathrm{D}, \mathrm{R}, \mathrm{A}$ et $\mathrm{T})$ ont été déjà préparées lors de l'élaboration des cartes DRASTIC. La carte relative au paramètre occupation des sols (OS) est la carte du facteur d'occupation des sols LU. Elle a été préparée en se basant sur la carte numérique d'occupation des sols du gouvernorat de Bizerte (CRDA de Bizerte, 1999). La carte de vulnérabilité SI obtenue (Figure 5) montre l'existence de trois degrés de vulnérabilité dans la zone d'étude : faible, moyen et élevé avec $20 \%$ des territoires à vulnérabilité faible, $73 \%$ à vulnérabilité moyenne, et $7 \%$ à vulnérabilité élevée.

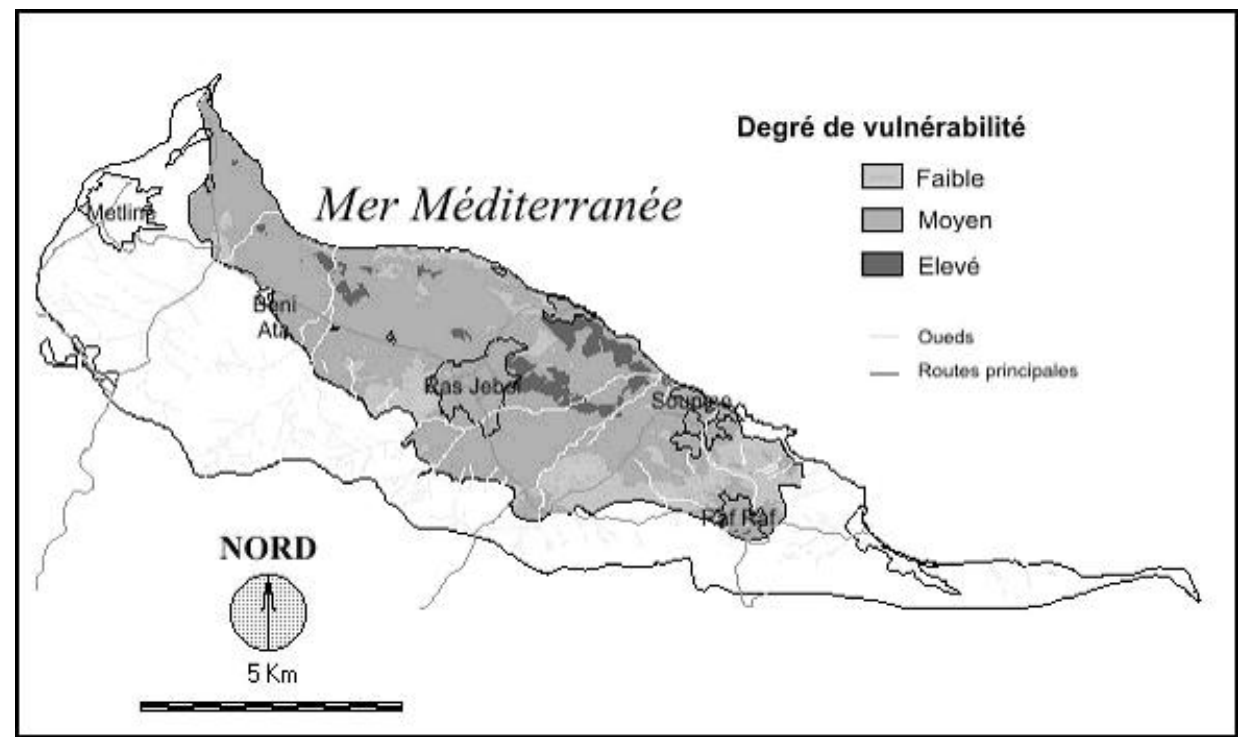

Figure 5 : Carte de vulnérabilité SI. SI vulnerability map. 


\section{COMPARASION DES CARTES DE VULNERABILITE OBTENUES}

Les résultas montrent que les cartes de vulnérabilité obtenues en se basant sur la méthode DRASTIC compte tenu des deux classifications d'ALLER et al. (1987) et d'ENGEL et al. (1996) sont différentes entre elles, et différentes par rapport à celles obtenues par les méthodes SI et SINTACS. D'autre part, les résultats obtenus par les méthodes SI et SINTACS ne différent pas significativement entre eux, notamment pour les zones à moyenne vulnérabilité, qui occupent la plus grande partie de la zone d'étude (Tableau 9).

En absence de validation des différentes méthodes on ne peut que constater que :

- La méthode DRASTIC, classée selon ALLER et al. (1987), donne des résultats largement différents des autres méthodes. Cette méthode ne permet de définir que deux classes de vulnérabilité : faible et moyenne.

- Les méthodes SI et SINTACS fournissent des résultats assez homogènes où prédomine nettement la classe de vulnérabilité moyenne $(80 \%$ de la surface totale pour la méthode SINTACS et $73 \%$ pour la méthode SI). Le taux de coïncidence relatif à la classe de vulnérabilité moyenne entre ces deux méthodes est de plus de $64 \%$.

- La méthode DRASTIC, selon ENGEL et al. (1996), donne des résultats qui se caractérisent par un taux de vulnérabilité moyenne de $48 \%$, avec un taux de coïncidence de $54 \%$ avec la classe de vulnérabilité moyenne de la méthode SINTACS et de $49 \%$ avec la classe de vulnérabilité moyenne de la méthode SI. La méthode DRASTIC, selon Engel et al., tend à augmenter la superficie des zones à vulnérabilité faible au détriment de la classe moyenne par rapport à la méthode DRASTIC selon ALLER et al., 1987

\section{CONCLUSIONS}

La comparaison des cartes de vulnérabilité à la pollution obtenues selon les méthodes DRASTIC (classification d'ALLER et al., 1987 et d'ENGEL et al., 1996), SINTACS et SI, montre que les résultats les plus proches sont ceux issus de la méthode SINTACS et SI, versions modifiées de la méthode DRASTIC adaptées au climat méditerranéen, climat régnant dans la zone d'étude. La carte de vulnérabilité DRASTIC, standard classique selon ALLER et al. (1987), donne, quant à elle, des résultats largement différents des autres méthodes.

Une validation de ces différentes méthodes de vulnérabilité utilisées mériterait d'être effectuée dans la région en établissant une comparaison des résultats qualitatifs et quantitatifs de la vulnérabilité avec les analyses de la qualité chimique des eaux.

Les résultats obtenus par ces méthodes paramétriques méritent également d'être comparés avec les résultats d'autres méthodes qui peuvent être appliquées dans la zone

Tableau 9: Comparaison des surfaces occupées par les différents degrés de vulnérabilité déterminés selon les méthodes.

Table 9: $\quad$ Percentage of surface occupied by the different vulnerability degrees according to the methods.

\begin{tabular}{|c|c|c|c|c|}
\hline $\begin{array}{l}\text { Méthode d'étude } \\
\text { Degré } \\
\text { de vulnérabilité }\end{array}$ & $\begin{array}{c}\text { DRASTIC, } \\
\text { (classification d'Aller } \\
\text { et al., 1987) }\end{array}$ & $\begin{array}{c}\text { DRASTIC, } \\
\text { (classification } \\
\text { d'Engel et al., 1996) }\end{array}$ & SINTACS & SI \\
\hline Faible & $77 \%$ & $46 \%$ & $19 \%$ & $20 \%$ \\
\hline Moyen & $23 \%$ & $48 \%$ & $80 \%$ & $73 \%$ \\
\hline Élevé & - & $6 \%$ & $1 \%$ & $7 \%$ \\
\hline Très élevé & - & - & - & - \\
\hline
\end{tabular}


d'étude. Parmi elles, on peut citer la méthode géophysique d'ANDRADE-AFONSO et al. (1998) développée au Portugal et qui peut être appliquée dans la zone étudiée en géophysique par ENNABLI (1969) et ESSAYEH (1996). Cette méthode permet de délimiter les zones à forte et faible vulnérabilité à la pollution, en se basant sur la détection des zones fracturées et sur la localisation et la quantification des discontinuités.

\section{RÉFÉRENCES BIBLIOGRAPHIQUES}

ADDED A., M.H. HAMZA (1999). Evaluation of the vulnerability in Metline aquifer (Northeast of Tunisia). ESRI user conference. San Diego, États-Unis.

ALLERL., T.BENNET,J.H.LEHR, R.J. PETTY, G. HACKET (1987). DRASTIC : A standardised system for evaluating ground water pollution potential using hydrogeologic settings (EPA 600/2-87). Environmental Research Laboratory, Office of Research and Development, U.S. Environmental Protection Agency, États-Unis, 455 p.

ANDRADE-AFONSO A.R., R.M.D. GONÇALVES, L.A. MENDES VICTOR (1998). A geofísica na detecção e parametrização de aquíferos - Algumas aplicaçóes. Lisboa, $4^{\circ}$ Congresso da Água da APRH, Lisbonne, Portugal.

ASB : Arrondissement des Sols de Bizerte (1997-2000). Comptes rendus d'études pédologiques établis à Metline, Ras Jebel et Raf Raf. Tunisie, $22 \mathrm{p}$.

BATISTA S. (2004). Exposição da água subterrânea a pesticidas e nitratos em ecossistemas agrícolas do Ribatejo e Oeste e da Beira Litoral. Tese de doutoramento em engenharia agronómica, Instituto superior de agronomia, Lisboa, Portugal, 19 p.

BUROLLET P.F. (1951). Étude géologique des bassins Mio-Pliocènes du Nord Est de la Tunisie. Ann. Mines Géol., 7, 82 p. + annexes + cartes $1 / 50.000$.

CRDA de Bizerte (1999). Carte numérique d'occupation des sols du gouvernorat de Bizerte au 1/25.000, Édition ministère de l'Agriculture, Tunis, Tunisie.

CIVITA M. (1994). La carte della vulnerabilità degli acquiferi all'inquiamento: Teoria e pratica. PITAGORA (Éditeurs), Bologna, Italie, $325 \mathrm{p}$.

DGRE (Direction Générale des Ressources en Eau) (1991-2004). Ann. piézom. Tunisie, Tunis, Tunisie.
EL GHALI A., N. BEN AYED (2000). Carte géologique au 1/50.000 de Metline. Publications du Service Géologique de Tunisie, Tunis, Tunisie.

ENGEL B.A., K.C.S. NAVULUR, B.S. COOPER, L. HAHN (1996). Estimating groundwater vulnerability to non-point source pollution from nitrates and pesticides on a regional scale. Int. Assoc. Hydrol. Sci., 235. 521-526.

ENGLAND C.B. (1973). Relative leaching potentials estimated from the hydrologic soil groups. Water Resour. Bull., 9, 590-597.

ENNABLI M. (1969). Étude hydrogéologique de la plaine de Ras Jebel. Rapport interne DGRE, réf. 7/57, Tunis, Tunisie, $134 \mathrm{p}+$ annexes.

ESSAYEH F. (1996). Apport de la méthode de prospection électrique à l'étude des problèmes d'intrusion marine dans la plaine de Metline Ras Jebel Raf Raf. DEA, Univ. Tunis II, Faculté des sciences, Tunis, Tunisie, $100 \mathrm{p}+$ annexes.

EUROPEAN COMMUNITY (1993). Corine land cover. Guide technique. Office des Publications Officielles des Communautés Européennes, Série environnement, sécurité nucléaire et protection civile, $144 \mathrm{p}$.

FOSTER S.S.D. (1987). Fundamental concepts in aquifer vulnerability, pollution risk and protection strategy. Dans : Vulnerability of soil and groundwater to pollutants, 38, W. VAN DUIJVENBOODEN, H.G. VAN WAEGENINGH (Éditeurs) TNO Committee on Hydrological Research, La Hague, Pays-Bas, pp. 69-86.

FOURNET A., A. MOURI (1990). Reconnaissance pédologique des périmètres d'irrigation de Beni Ata et de Chaab Eddoud. Rapport interne, Direction des Sols, Édition direction des sols, Tunisie.

FRANCES A., E. PARALTA, J. FERNANDES, L. RIBEIRO (2002). Development and application in the Alentejo region of a method to assess the vulnerability of groundwater to diffuse agricultural pollution: the susceptibility index. Dans : RIBEIRO L. (Éditeur) FGR'01 - $3^{\text {rd }}$ International conference on future groundwater resources at risk, p. 35-44, CVRM (Éditeur), Lisbonne, Portugal.

HAMZA M.H., A. ADDED, A. BEN MAMMOU, S. ABDELJAOUED, R. RODRÍGUEZ (2004). Évaluation de la vulnérabilité à la pollution potentielle par les pesticides, de la nappe côtière alluvionnaire de la plaine de Metline-Ras Jebel-Raf Raf, Nord-Est tunisien, selon la méthode DRASTIC appliquée par les systèmes 
d'information géographique. Houille Blanc. 2004, 86-94. http://dx.doi.org/10.1051/lhb:200405011

HAMZA M.H., A. ADDED, R. RODRIGUEZ, S. ABDELJAOUED, A. BEN MAMMOU (2007). A GISbasedDRASTICvulnerability and net recharge reassessment in an aquifer of a semi-arid region (Metline-Ras Jebel-Raf Raf aquifer, Northern Tunisia). J. Environ. Manage., 84, 12-19, http://dx.doi.org/10.1016/j.jenvman.2006.04.004

INM (Institut de la Météorologie Nationale) (1991-2004). Tableaux climatologiques mensuels, station Bizerte-Sidi Ahmed, Tunisie.

MANSOUR H. (1988). Carte pédologique de Beni Ata, Ras Jebel et Raf Raf au 1/12.500, DIRECTION DES SOLS DE TUNISIE (Éditeurs), Tunisie,

OLIVEIRA M., J.P. LOBO-FERREIRA (2005). Análise de sensibilidade de aplicação de métodos indexados de avaliação da vulnerabilidade à poluição de águas subterrâneas in Ribeiro L., Peixinho de Cristo F., Andreo B. \& SanchezVila $X$. As águas subterrâneas no sul da península Ibérica, APRH (Éditeurs), Lisbonne, Portugal, 239-252.

OTC (Office de la Topographie et de la cartographie) (1981). Carte topographique de la Tunisie au 1/25.000. Feuilles de Metline S.O., Metline S.E. et Ghar El Melh N.E., Tunisie.

RIBEIRO L. (2000). Desenvolvimento de um indice para avaliar a susceptibilidade dos aquíferos à contaminação. Nota interna, (não publicada), ERSHA-CVRM, 8 p.

RODRÍGUEZ R., R. REYES, J. ROSALES, J. BERLIN, J.A. MEJIA, A. RAMOS (2001). Estructuracion de mapas tematicos de indices de vulnerabilidad acuifera de la mancha urbana de Salamanca Gto., Municipio de Salamanca. CEAG, IGF-UNAM. Technical Report, Mexico, Mexique, $120 \mathrm{p}$.

STIGTER T.Y., L. RIBEIRO, A.M.M. CARVALHO DILL (2006). Evaluation of an in trinsic and a specific vulnerability assessment method in comparison with groundwater salinisation and nitrate contamination levels in two agricultural regions in the south of Portugal, Hydrogeol. J., 14, 79-99, http://dx.doi.org/10.1007/s10040-004-0396-3.

VRBA J., A. ZOPOROZEC (1994). Guidebook on mapping groundwater vulnerability, in international contributions to hydrogeology, 16, I. A. HYDROGEOLOGISTS (Éditeurs), $131 \mathrm{p}$. 